\title{
METHODS FOR COMPUTING AND INTERCOMPARING RADIATION DATA
}

\author{
By W. W. Coblentz
}

\section{INTRODUCTORY STATEMENT}

This paper gives a simple method for computing spectral energy curves, using the Planck formula. In order to facilitate the computations, a table of values of $\log \left(e^{u}-1\right)$ is given. Such data do not appear to have been published heretofore. By means of this table, a table of logarithms, and a slide rule, one can compute a complete spectral energy curve in an hour.

The paper gives also a chart for intercomparing the thermal radiation constants, with similar data which may be obtained indirectly from theoretical considerations, using Planck's universal constant, $h$, and data obtained from ionization-potential, photoelectric, and X-ray work. Of course it is to be understood that whatever the true (theoretical) value of the constant, $h$, and the spectral radiation constant, $c_{2}$, may be, the value of $c_{2}$, as obtained from measurements on black-body radiators should be used in optical pyrometry and radiation work.

During the past few years the writer ${ }^{1}$ has made frequent use of this table in computing spectral energy data and has found it sufficiently extensive for ordinary use. The chart is the outcome of numerous intercomparisons ${ }^{2}$ of spectral radiation data with similar data resulting from photoelectric and other experiments, supplemented by the very complete computations and tabulations made by Dushman. ${ }^{3}$

\section{COMPUTATION OF SPECTRAL RADIATION DATA}

The Planck radiation formula, as usually written, is

$E_{\lambda}=c_{1} \lambda-5\left(e c_{3} \lambda \mathrm{T}-\mathrm{I}\right)-1$

The function, $e^{u}-1$, does not appear to have been tabulated heretofore. Some years ago a system of computation was

\footnotetext{
1 Bulletin, Bureau of Standards, 13, p. 47r, r9r6.

2 Coblentz, Phys. Rev., 32, p. 613, $191 x$ (On the Elementary Electrical Charge); also Bulletin, Bureau of Standards, 12 , p. 553 , 1916.

${ }^{3}$ Dushman, Gen. Elect. Rev., 18, p. I167, I915. 150057-20 
adopted whereby the value of $\lambda$ was so chosen that the exponent $u=c_{2} / \lambda T$ resulted in a number which is given in exponential tables ${ }^{4}$ of $e^{u}$. This, of course, is possible, whatever values of $c_{2}$ may be used. Hence, the present table is of permanent value. In view of the uncertainty of the exact value of $c_{2}$, the time does not yet seem ripe for tabulating $E_{\lambda}$, for different temperatures (say, in steps of $50^{\circ}$ ) and wave lengths.

By the system of calculation herein employed, spectral intensity data may be quickly computed. For this purpose the value of $\log c_{1}=5.00000$ is employed, the resulting values of $E_{\lambda}$ being, of course, in arbitrary units. The Planck formula then becomes

$\log E_{\lambda}=\log k-5 \log \lambda$

in which values of $\log k=\log c_{1}-\log \left(e^{u}-I\right)$ are given in the fourth column of Table $I$.

As already mentioned, the usefulness of the method lies in selecting the exponent

$u=c_{2} / \lambda T$

so that values of $u$ may be taken from the table. The value of $\lambda$ usually comes out some uneven number, but this is unimportant.

Example.-The following data are given as an illustration of the computation ${ }^{5}$ of the isothermal spectral energy curve for

Here

$$
T=\mathrm{I} 596^{\circ} \mathrm{K} \text {, using } c_{2}=\mathrm{I} 435 \mathrm{O} \text {. }
$$

$$
u=c_{2} \lambda \lambda T=\frac{8.99}{\lambda}
$$

From Table $\mathbf{I}$, for

$$
\begin{gathered}
u=8.0, \lambda=\mathrm{I} . \mathrm{I} 23 \mu ; \\
\log k=\mathrm{I} .52579 \\
\frac{5 \log \lambda=.25 \mathrm{I} 90}{\log E_{\lambda}}=\mathrm{I} .273^{89} \\
E=\mathrm{I} 8.79 \text { at } \lambda=\mathrm{I} . \mathrm{I} 23 \mu .
\end{gathered}
$$


TABLE 1

\begin{tabular}{|c|c|c|c|}
\hline$u$ & $e^{\mathrm{u}}$ & $\log _{10}\left[e^{u}-1\right]$ & $\begin{array}{c}\log k= \\
5-\log \left[e^{u}-1\right]\end{array}$ \\
\hline 0.10 & 1. 10517 & ‥ 021932 & 5. 97807 \\
\hline .15 & 1. 16183 & '̄. 209069 & 5. 79094 \\
\hline .20 & 1.22140 & I. 345177 & 5.65483 \\
\hline .25 & 1. 28403 & 1. 453357 & 5. 54665 \\
\hline .30 & 1. 34986 & 1.. 543894 & 5. 45611 \\
\hline 0.35 & 1. 41907 & 1. 622286 & 5. 37772 \\
\hline .40 & 1. 49183 & $\overline{1} .691815$ & 5. 30819 \\
\hline .45 & 1. 56831 & 1.. 754586 & 5. 24542 \\
\hline .50 & 1. 64872 & I. 812057 & 5. 18795 \\
\hline .55 & 1.73325 & 1. 865254 & 5. 13475 \\
\hline 0.60 & 1.82212 & I. 914935 & 5. 08507 \\
\hline .65 & 1. 91554 & 1. 961677 & 5.03833 \\
\hline .70 & 2.01375 & 0.005926 & 4. 99407 \\
\hline .75 & 2. 11700 & .048053 & 4. 95195 \\
\hline .80 & 2. 22554 & .088317 & 4.91169 \\
\hline 0.85 & 2. 33965 & .126971 & 4. 87303 \\
\hline .90 & 2. 45960 & .164234 & 4. 83577 \\
\hline .95 & 2. 58571 & .200221 & 4. 79978 \\
\hline 1.00 & 2. 71828 & .235099 & 4. 76491 \\
\hline .05 & 2.85765 & .268964 & 4.73104 \\
\hline 1. 10 & 3. 00417 & .301935 & 4. 69808 \\
\hline .15 & 3. 15819 & . 334089 & 4. 66591 \\
\hline .20 & 3. 32012 & .365509 & 4. 63449 \\
\hline .25 & 3. 49034 & . 396257 & 4. 60374 \\
\hline .30 & 3. 66930 & .426397 & 4. 57361 \\
\hline 1.35 & 3. 85743 & .455976 & 4. 54403 \\
\hline .40 & 4.05520 & .485039 & 4. 51497 \\
\hline .45 & 4. 26312 & .513633 & 4. 48637 \\
\hline .50 & 4. 48169 & .541789 & 4. 45822 \\
\hline .55 & 4. $71 \quad 147$ & .569546 & 4. 43046 \\
\hline 1.60 & 4. 95303 & .596929 & 4. 40308 \\
\hline .65 & 5. 20698 & .623970 & 4. 37603 \\
\hline .70 & 5. 47395 & .650691 & 4. 34931 \\
\hline .75 & 5. 75460 & .677114 & 4. 32289 \\
\hline .80 & 6.04965 & .703261 & 4. 29674 \\
\hline
\end{tabular}


TABLE 1-Continued

\begin{tabular}{|c|c|c|c|}
\hline$u$ & $e^{v}$ & $\log _{10}\left[e^{u}-1\right]$ & $\begin{array}{c}\log k= \\
5-\log \left[e^{\mathrm{u}}-1\right]\end{array}$ \\
\hline 1.83 & 6. 23389 & .718825 & 4. $28 \quad 118$ \\
\hline .85 & 6. 35982 & .728150 & 4. 27185 \\
\hline .90 & 6. 68589 & .754798 & 4. 24521 \\
\hline .93 & 6.88951 & .770079 & 4. 22992 \\
\hline .95 & 7.02869 & .780223 & 4. 21978 \\
\hline 2.00 & 7.38906 & .805437 & 4. 19456 \\
\hline .10 & 8. 16617 & .855287 & 4. 14471 \\
\hline .20 & 9.02501 & .904446 & 4. 09555 \\
\hline .30 & 9.97418 & .952995 & 4. 04701 \\
\hline .40 & 11.0232 & 1. 001005 & 3. 99899 \\
\hline 2. 50 & 12.1825 & 1. 048529 & 3.95148 \\
\hline .60 & 13.4637 & 1.095647 & 3. 90435 \\
\hline .70 & 14.8797 & 1. 142386 & 3. 85761 \\
\hline .80 & 16.4446 & 1. 188779 & $3.81 \quad 122$ \\
\hline .90 & 18. 1741 & 1. 234875 & 3. 76513 \\
\hline 3.00 & 20.0855 & 1. 280705 & 3. 71929 \\
\hline .10 & 22.1980 & 1. 326293 & 3. 67371 \\
\hline .20 & 24.5325 & 1. 371668 & 3. 62833 \\
\hline .30 & 27.1126 & 1.416851 & 3. 58315 \\
\hline .40 & 29.9641 & 1.461859 & 3. 53815 \\
\hline 3. 50 & 33. 1155 & 1. 506714 & 3.49329 \\
\hline .60 & 36.5982 & 1. 551428 & 3. 44857 \\
\hline .70 & 40.4473 & 1. 596017 & 3. 40398 \\
\hline .80 & 44.7012 & 1.640493 & 3.35951 \\
\hline .90 & 49.4024 & 1.684867 & 3. 31513 \\
\hline 4.00 & 54.5982 & 1. 729150 & 3. 27085 \\
\hline .10 & 60.3403 & 1. 773349 & 3. 22664 \\
\hline .20 & 66.6863 & 1. 817541 & 3. 18246 \\
\hline .30 & 73. 6998 & 1.861532 & 3. 13846 \\
\hline .40 & 81.4509 & 1.905531 & 3.09446 \\
\hline 4.50 & 90.0171 & 1. 949474 & 3. 05053 \\
\hline .60 & 99.4843 & 1.993367 & 3.00663 \\
\hline .70 & 109. 947 & 2. 037215 & 2. $96 \quad 278$ \\
\hline .80 & 121. 510 & 2. 081023 & 2.91898 \\
\hline .90 & 134. 290 & 2. 124798 & 2. 87520 \\
\hline
\end{tabular}


TABLE 1-Continued

\begin{tabular}{|c|c|c|c|}
\hline$u$ & $e^{\mathrm{u}}$ & $\log _{10}\left[e^{\mathrm{u}}-1\right]$ & $\begin{array}{c}\log k= \\
5-\log \left[e^{\mathrm{u}}-1\right]\end{array}$ \\
\hline 5. 00 & 148. 413 & 2. 168536 & 2. 83146 \\
\hline .10 & 164. 022 & 2. 212246 & 2. 78775 \\
\hline .20 & 181. 272 & 2. 255928 & 2. 74407 \\
\hline .30 & 200. 337 & 2. 299588 & 2. 70041 \\
\hline .40 & 221. 406 & 2. 343223 & 2.65678 \\
\hline 5.50 & 244. 692 & 2. 386841 & 2. 61316 \\
\hline .75 & 314. 191 & 2. 495809 & 2. 50419 \\
\hline 6.00 & 403. 429 & 2. 604689 & 2. 39531 \\
\hline .50 & 665. 142 & 2. 822261 & 2. 17774 \\
\hline 7.00 & 1096.63 & 3. 039664 & 1.96034 \\
\hline 7. 50 & 1808.04 & 3. 256959 & 1. 74304 \\
\hline 8.00 & 2980.95 & 3. 474209 & 1. 52579 \\
\hline 8. 50 & 4914.77 & 3. 691414 & 1. 30859 \\
\hline 9.00 & 8103.08 & 3. 908596 & 1.09140 \\
\hline 9.50 & 13359.7 & 4. 125770 & .87423 \\
\hline 10.0 & 22026.5 & 4. 342926 & .65707 \\
\hline 11.0 & 59874.1 & 4. 777232 & .22277 \\
\hline 12.0 & 162755. & 5.211532 & I. 78847 \\
\hline 13.0 & 442413. & 5.645828 & I. 35417 \\
\hline 14.0 & 1202604. & 6.080123 & I. 91988 \\
\hline
\end{tabular}

It is of course to be understood that after computing these data they must be multiplied by a factor to superpose them upon the observed spectral energy curve. This, as well as the determination of the different values of $u$, is easily accomplished with a slide rule.

\section{INTERCOMPARISON OF RADIATION AND OTHER DATA}

From Planck's radiation theory we have the following relations:

$$
\begin{aligned}
& c_{2}=c h k^{-1}=4.965 \mathrm{I} \lambda_{\mathrm{m}} T . \\
& \sigma=\frac{a c}{4}=\frac{\mathrm{I} 2 \pi \times \mathrm{I} .0823 k^{4}}{c^{2} h^{3}} \ldots \\
& \lambda_{\mathrm{m}} T=\frac{c h}{4.965 \mathrm{I} k} \ldots \ldots \ldots \ldots \\
& k=\frac{e R}{c F} \ldots \ldots \ldots \ldots
\end{aligned}
$$


In these equations $h$ is Planck's universal constant or constant of action; $k$ is the Boltzmann gas constant, $k=1.372 \times 10^{-16} \mathrm{erg}$. $\operatorname{deg}^{-1} ; c$ is the velocity of light, $c=2.9986 \times 10^{10} \mathrm{~cm}$. sec. ${ }^{-1} ; F$

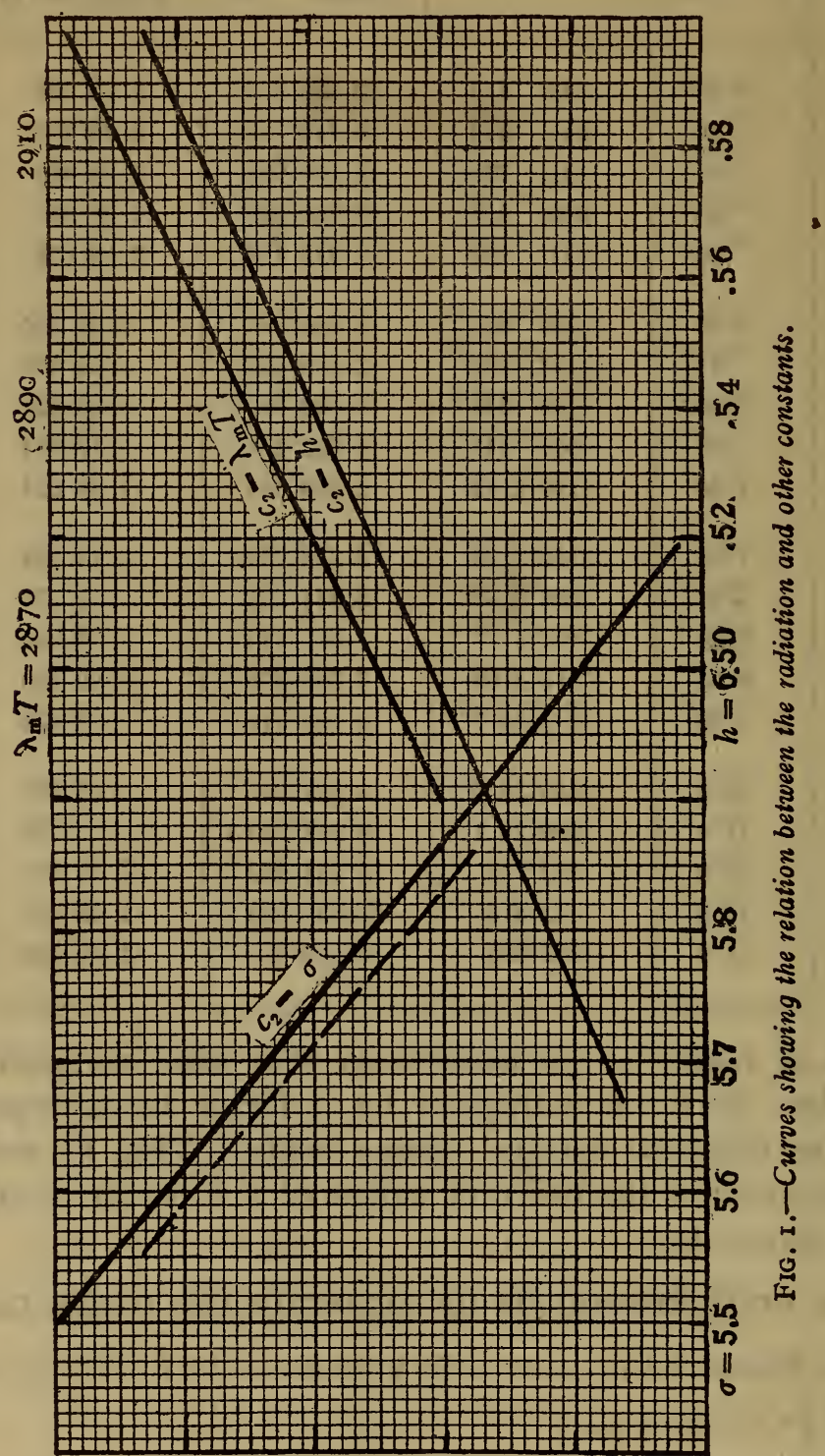

is the Faraday constant, $F=96500$ coulombs; $R$ is the absolute gas constant, $R=83 \mathrm{I} .5 \mathrm{erg}$. $\mathrm{deg}^{-1}$; and $e$ is the unit electric charge, $e=4.774 \times \mathrm{rO}^{-10}$ e. s. u. From equation (7) it may be 
noticed that a change in the value of $e$ affects the value of $c_{2}$ directly, while the value of $\sigma$ is affected by $e^{4}$.

The data computed from the above-mentioned constants and formulæ are illustrated in Fig. $I$, from which it is an easy matter to compare experimental data. For example, the writer's ${ }^{\circ}$ value of the coefficient of total radiation is $\sigma=(5.72 \pm 0.012) \times 10^{-12}$ watt. $\mathrm{cm}^{-2}$. deg-4. This indicates a value of $c_{2}=14320$ micron degrees and a value of $h=6.55 \times 1 \mathrm{O}^{-27} \mathrm{erg} \mathrm{sec}$. The value of $h$, determined by Blake and Duane ${ }^{7}$ by $\mathrm{X}$ rays, is $h=6.555 \times 1 \mathrm{I}^{-27}$ erg sec.; or an indicated value of $c_{2}=14330$ micron degrees, which is close to experimental determinations of this constant.

Using the early determinations of $c_{2}$ and $\sigma$ by Lummer and Pringsheim, and by Kurlbaum, and the above-mentioned relations in his radiation theory, Planck $^{8}$ deduced a value of $e=$ $4.69 \times 10^{-10}$ e. s. $\mathrm{u}$. for the elementary charge of an electron. He calls attention to the fact that the value of this constant will depend upon the accuracy of the experimental determination of the constants of radiation, which in the meantime have been found quite different from the values used by him.

In the meantime the unit electric charge has been determined with high precision by Millikan, and recent writers have been computing the radiation and other constants, using the value of $e=4.774 \times 1 \mathrm{IO}^{-10}$ e. s. 1. As shown by the dotted, $c_{2}, \sigma$-curve in Fig. I, a change of about $0 . \mathrm{I}$ per cent in $e$ (using $e=4.777$ as found in earlier experiments-I9I2) has an appreciable effect upon, and happens to bring a closer agreement between, the writer's values of $c_{2}$ and $\sigma$; though no particular significance is to be attached to this fact.

In view of the fact that several writers ${ }^{10}$ have expressed the opinion that the radiation constants $c_{2}$ and $\sigma$ could be derived more accurately from determinations of $h$ by photoelectric and other data, it is important to emphasize that whatever the theoretical value of $c_{2}$ may be, the value of $c_{2}$ as determined on the best black bodies that can be constructed, using accepted experimental methods, is the one to be used in optical pyrometry and radiation work.

\footnotetext{
6 Proc. Nat. Acad. Sci., 3, p. 504, 1917.

7 Blake and Duane, Phys. Rev. (2), 10, p. 624, 1917.

8 Planck, Vorlesungen ï Warmestrahlung, p. $163 ; 1906$.

9 Millikan, Proc., Nat. Acad. Sci., 3, p. 23I; 1917.

${ }_{10}$ Millikan, Phys. Rev., (2) 7, p. 378; r916. Dellinger, Bulletin, Bureau of Standards, 13, p. 543; 1916.
} 
From a recent recalculation and intercomparison by Birge ${ }^{11}$ of the data on $c_{2}, \sigma$, and $h$, as determined by radiometric, photoelectric, $\mathrm{X}$ rays, and ionization potential measurements, it appears that the value of $h$, computed from radiometric data, compares favorably with that obtained by more direct measurement. The outstanding disagreement between all the observed and computed data appears to be of the order of 2 to 3 parts in Iooo, whatever the method of experimentation. This is a very close agreement, considering the variety of the data and the difficulties involved in making the experiments. It seems to indicate something more than a fortuitous relation between properties of matter.

\section{SUMMARY}

In this paper a simple method is given for computing spectralenergy curves, using the Planck formula. For this purpose a table of values of $\log \left(e^{u}-1\right)$ is given.

The paper gives also a chart for the intercomparison of thermal radiation constants with similar data, obtained indirectly from ionization potential, photoelectric, and X-ray measurements.

Washington, August 6, I9I9. 